\title{
Profile of Gynaecological Malignancies in Alex Ekwueme Federal University Teaching Hospital, Abakaliki, Southeastern Nigeria
}

\author{
Ayodele A. Olaleye, Johnbosco I. Nwafor*, Alfred N. Adiele, Chuka N. Obi, Sunday U. Asogwa, \\ Chukwunenye C. Ibo, Darlington-Peter C. Ugoji, Ikenna C. Ebere \\ Department of Obstetrics and Gynaecology, Alex Ekwueme Federal University Teaching Hospital, Abakaliki, Nigeria \\ Email: *nwaforjohnbosco97@gmail.com
}

How to cite this paper: Olaleye, A.A., Nwafor, J.I., Adiele, A.N., Obi, C.N., Asogwa, S.U., Ibo, C.C., Ugoji, D.-P.C. and Ebere, I.C. (2019) Profile of Gynaecological Malignancies in Alex Ekwueme Federal University Teaching Hospital, Abakaliki, Southeastern Nigeria. International Journal of Clinical Medicine, 10, 428-438. https://doi.org/10.4236/ijcm.2019.109036

Received: July 27, 2019

Accepted: September 15, 2019

Published: September 18, 2019

Copyright $\odot 2019$ by author(s) and Scientific Research Publishing Inc. This work is licensed under the Creative Commons Attribution International License (CC BY 4.0).

http://creativecommons.org/licenses/by/4.0/

\begin{abstract}
Background: Comprehensive estimates of the incidence of gynaecological malignancies reported from Nigeria are very limited due to limitation in record maintenance. Female cancer is a public health problem the world over. The malignancies of the female genital organs are major causes of morbidity and mortality which necessitates data for policy formulation and health planning. Aim: To establish the profile of gynaecologic malignancies reported in our centre, with reference to incidence, histological subtypes and frequency of involvement at various sites and to compare the procured data with those from other national and international centers. Materials and Method: In this descriptive retrospective study, the records pertaining to all the pathological specimens categorized as gynaecological malignancies from January 1st, 2013 to December 31st, 2015 at our facility were studied and compared with the available international data. Data analysis was done using Epi Info software version 7.2.1 (CDC Atlanta Georgia). Result: Two hundred and eleven (211) cases of gynaecological malignancies were seen during the study period and this constituted $13.4 \%$ of gynaecological admissions. Cervical malignancies were the commonest constituting $49.2 \%$ of all gynaecological malignancies followed by ovarian malignancies $(27.8 \%)$, endometrial cancers (11.9\%), vulva $(5.6 \%)$, choriocarcina $(3.9 \%)$ and vagina $(1.6 \%)$. The data obtained was compared with data from Surveillance, Epidemiology and End Result (SEER) programme in the United States and European Union. All malignancies except cervical cancers affected a younger age group at our centre. Cervical cancers presented at a relatively more advanced stage, Ovarian cancers were more localized, whereas uterine, vulva and vaginal cancers presented at a similar stages as compared to Western data. Conclusion: This study presents a composite data of Gynaecological malignancies from Southeast Nigeria. Ad-
\end{abstract}


vanced stage of presentation of cervical cancers suggests lacunae in screening programmes available. Compared with Western data, ovarian malignancies were more localized at presentation for which environmental or genetic factors may be responsible.

\section{Keywords}

Profile, Gynaecological, Malignancies, Abakaliki, Nigeria

\section{Introduction}

Gynaecological cancers form a huge burden of morbidity and mortality around the world [1]. Data available from various centres worldwide are indicative of vast regional variability in incidence, common site of occurrence, age and stage of presentation [2]. While information on such issue is readily available from the developed world, composite data from Nigeria are deficient [3].

Cancer is a public health problem for the world over [4]. Malignancies of the female genital organs are major causes of morbidity and mortality [5]. This is particularly so in the developing countries where there are poor awareness and late presentation [6]. Worldwide, gynaecological cancers account for about $10 \%$ of new cancer cases in women and $12 \%$ of cancer deaths [6]. The incidence of gynaecological malignancies has decreased drastically in developed countries due to organized screening programs [7]. However, in Sub-Saharan Africa, squamous cell carcinoma of the cervix accounts for the largest proportion of genital tract cancers [8].

Endometrial carcinoma has become the most common gynaecological malignancies in North America and Northern Europe while it is less commonly seen amongst black women [9]-[20]. Ovarian cancer is one of the common gynaecological malignancies with considerable geographical variation, the incidence in the highest risk countries being 5 times greater than that in the lowest risk countries [10]. It is the leading cause of mortality of female gynaecological cancers and ranks seventh as the most common cancer worldwide [11]. The increasing life expectancy has led to increase in its burden exponentially now even in developing countries but limited knowledge is there about the pattern of ovarian tumours [12] There is scanty literature on the current state of ovarian cancer in Nigeria. Cervical cancer is the most common cause of cancer-related death in the developing countries because of poor cervical cancer screening programs in these countries [12] [13] [14] [15]. Vulval and vaginal cancers are rare with peak incidence in the 6th decade of life [16]-[25].

There has been limited recent information on the profile of gynaecological cancers in Abakaliki, hence the decision to carry out this study to determine the profile of gynaecological malignancies in Alex Ekwueme Federal University Teaching Hospital Abakaliki, as this could have significant implications on health planning and policy decisions. Therefore, the aim of this study was to establish 
the profile of gynaecologic malignancies managed in our centre, with reference to sociodemographical data, histological subtypes and frequency of involvement at various sites and to compare the procured data with those from other national and international centres.

\section{Materials and Method}

Study Area: Alex Ekwueme Federal University Teaching Hospital (formerly known as Federal Teaching Hospital) Abakaliki is a tertiary hospital within Abakaliki metropolis. The hospital is made up of ten clinical departments; Obstetrics and Gynaecology, Paediatrics, Medicine, Surgery, Psychiatry, Community Health, Family Medicine, Ophthalmology, ENT and Anesthesiology. Department of Obstetrics and Gynaecology is 1 of the 10 clinical departments in the hospital, it has ten teams with each comprising consultants, senior registrars, registrars, senior house officers and house officers. The department runs gynaecological clinics, preconception, antenatal, intrapartum, and postnatal services. It is also a referral centre to the surrounding maternities and hospitals and also receives referral from surrounding states; Cross Rivers, Enugu, and Benue states. Patients with gynaecological malignancies are referred from all these places to the hospital.

Study Design: This was a retrospective descriptive study of the profile of gynaecological malignancies in Alex Ekwueme Federal University Teaching Hospital Abakaliki over a three year period. The case files of all women managed for gynaecological malignancies at the hospital from 1st January, 2013 to 31st December, 2015 was retrieved from the Medical Records Department, and information was also obtained from admission records in Gyaenecological ward, record of Operations at the Theatre, and also from the record of histological report of samples of all cases of gynaecological malignancies analyzed at the Histopathology department of the hospital. The inclusion criterion was women with diagnosis any of the type of gynaecological cancers confirmed by histology.Data were extracted pertaining socio-demographic and gynaecological characteristics including age, parity, and occupational status, presenting symptoms, risk factors for malignancy, organ of involvement, provisional diagnosis and histopathological diagnosis.

Data Analysis: Data collection was done using a pre-designed proforma. Data analysis was done using Epi Info software (7.2.1 CDC Atlanta Georgia). The results were expressed as frequency tables, percentages, mean and standard deviation. Associations between categorical data were analyzed using $\mathrm{X}^{2}$, with a p-value of 0.05 considered statistically significant.

Ethical Consideration: Permission to carry out this research was sought and obtained from the Research and Ethical Committee of the Alex Ekwueme Federal University Teaching Hospital Abakaliki.

\section{Results}

During the study period, 1570 women were admitted into the gynaecological 
ward. Two hundred and eleven (211) had gynaecological cancers from the admission records. This gave the proportion of gynaecological cancer of $13.4 \%$ of gynaecological admissions. One hundred and sixty two (162) case notes were retrieved giving a retrieval rate of $76.78 \%$ and 126 of these retrieved case notes had complete records including histological diagnoses. Percentage of case notes containing all information was $77.78 \%$. The sites of involvement included the cervix (62), ovary (35), corpus uteri (15), vulva (7), choriocarcinoma (5) and vagina (2).

Table 1 showed the socio-demographic data of patients with gynaecological malignancies. The mean age of women who had cervical carcinoma (51.8 \pm 4.9 years) was higher than that of those with ovarian and endometrial cancer (50.3 \pm 6.1 years and $50.7 \pm 6.1$ years respectively) but was lower than those with vulva $(67.8 \pm 2.7$ years $)$ and vagina $(68.0 \pm 1.0$ years $)$ cancers. The mean age of patients with choriocarcinoma (31.7 \pm 3.2 years) was lowest. Half of cases of cervical cancer ( 31 out of 62 ) were aged $55-74$ years and one patient was aged 84 years. The same trend was observed in other gynaecological malignancies and the two cases of vaginal cancer were in $55-74$ years age group. Low frequencies of gynaecological malignancies were observed in patients between the ages of 15 years to 34 years ( 9 cases of cervical cancer, 6 cases of ovarian, 2 cases of endometrial cancers and 3 cases of choriocarcinoma). None of the patients between the ages of 15 to 54 years had either vulva or vagina cancer. The mean parity for patients with cervical cancer $(6.4 \pm 0.6)$ was higher than those of ovarian cancer $(3.8 \pm$ $1.2)$, endometrial cancer $(3.9 \pm 1.1)$, choriocarcinoma ( $3.5 \pm 2.3)$ and vulva cancer $(5 \pm 1)$. There is an increase in incidence of cervical cancer with increasing parity but this was not statistically significant $(P=0.2324)$. In patients with endometrial, ovarian, choriocarcinoma, vulva and vaginal carcinomas, there was no significant increase in incidence of cancers with increasing parity and the relationships were not statistically significant $(P>0.05)$. None of the patients seen used tobacco in any form.

As shown in Table 2 and Table 3, sixty two (49.2\%) patient had cervical cancer

Table 1. Socio-demographic characteristics of patients with gynaecological malignancies.

\begin{tabular}{|c|c|c|c|c|c|c|}
\hline Parameters & Cervical & Ovarian & Endometrial & Choriocarcinoma & Vulva & Vaginal \\
\hline \multicolumn{7}{|l|}{ Age } \\
\hline $15-35$ & 9 & 6 & 2 & 3 & 0 & 0 \\
\hline $35-54$ & 21 & 13 & 6 & 2 & 0 & 0 \\
\hline $55-74$ & 31 & 16 & 7 & 0 & 6 & 2 \\
\hline $75-84$ & 1 & 0 & 0 & 0 & 1 & 0 \\
\hline Mean Age & $51.8 \pm 4.9$ & $50.3 \pm 6.1$ & $50.7 \pm 6.3$ & $31.7 \pm 3.2$ & $67.8 \pm 2.7$ & $68.0 \pm 1.0$ \\
\hline \multicolumn{7}{|l|}{ Parity } \\
\hline 0 & 0 & 3 & 1 & 0 & 0 & 0 \\
\hline $1-4$ & 20 & 25 & 10 & 4 & 3 & 1 \\
\hline $5-10$ & 42 & 7 & 4 & 1 & 4 & 1 \\
\hline Mean Parity & $6.4 \pm 0.6$ & $3.8 \pm 1.2$ & $3.9 \pm 1.1$ & $3.5 \pm 2$ & $4.0 \pm 1.0$ & $9.0 \pm 1$ \\
\hline P Value & 0.2324 & 0.3208 & 0.6537 & 30.5432 & 0.7108 & 0.2978 \\
\hline
\end{tabular}


Table 2. Stage at presentation and Histological subtype of gynaecological malignancies (stage according to FIGO classification).

\begin{tabular}{|c|c|c|c|c|c|c|c|c|c|c|c|}
\hline \multirow[t]{2}{*}{ Stage } & \multicolumn{2}{|c|}{ Cervix } & \multicolumn{3}{|c|}{ Ovary } & \multicolumn{3}{|c|}{ Uterus } & \multirow{2}{*}{$\begin{array}{c}\text { Vulva } \\
\text { SCC }\end{array}$} & \multirow{2}{*}{$\begin{array}{c}\text { Vagina } \\
\text { SCC }\end{array}$} & \multirow[t]{2}{*}{ Total } \\
\hline & SCC & Adeno & Epith & Germ & Stroma & Epith & Mesen & Chorio & & & \\
\hline 1 & 13 & 3 & 3 & 4 & 2 & 1 & 2 & 2 & 0 & 0 & 30 \\
\hline 2 & 8 & 1 & 8 & 3 & 1 & 3 & 1 & 1 & 2 & 1 & 29 \\
\hline 3 & 18 & 1 & 11 & 1 & 0 & 6 & 0 & 1 & 2 & 1 & 41 \\
\hline 4 & 18 & 0 & 1 & 1 & 0 & 2 & 0 & 1 & 3 & 0 & 26 \\
\hline Total & & 62 & & 35 & & & 20 & & 7 & 2 & 126 \\
\hline
\end{tabular}

SCC $=$ squamous cell carcinoma; Adeno = adenocarcinoma; Epith = epithelial cancers; Mesen = mesenchymal cancer; Germ = germ cell cancers; chorio = choriocarcinoma.

Table 3. Sites of involvement and median age of patients compared with international data [2] [24] [25] [26] [27].

\begin{tabular}{ccccccccccc}
\hline Site & \multicolumn{2}{c}{ AEFUTHA } & \multicolumn{2}{c}{ SEER } & \multicolumn{2}{c}{ European Union } & Indonesia & \multicolumn{2}{c}{ India } \\
\hline & \% Cases & $\begin{array}{c}\text { Median } \\
\text { Age }\end{array}$ & \% Cases & $\begin{array}{c}\text { Median } \\
\text { Age }\end{array}$ & \% Cases & $\begin{array}{c}\text { Median } \\
\text { Age }\end{array}$ & \% Cases & $\begin{array}{c}\text { Median } \\
\text { Age }\end{array}$ & $\begin{array}{c}\text { \% Cases } \\
\text { Aedian } \\
\text { Age }\end{array}$ \\
\hline Cervix & 49.2 & 51 & 16.24 & 48 & 18.28 & NA & 75.00 & NA & 71.5 & 50 \\
Ovary & 27.8 & 45 & 26.95 & 63 & 36.34 & NA & 19.63 & NA & 15.11 & 45 \\
Uterus & 15.9 & 50 & 49.14 & 65 & 38.92 & NA & 4.2 & NA & 9.95 & 52 \\
Vulva & 5.6 & 68 & 4.67 & 68 & 5.16 & NA & 0.68 & NA & 2.7 & 60 \\
Vaginal & 1.6 & 74 & 1.48 & 68 & 1.29 & NA & 0.46 & NA & 0.7 & 50 \\
\hline
\end{tabular}

AEFUTHA = Alex Ekwueme Federal University Teaching Hospital, Abakaliki; NA = not available; SEER = surveillance, epidemiology and end result. Median age is expressed in years, $\%=$ percentage.

with a median age of 51 years and only 20 patients (32.3\% of cervical cases) presented at an early stage and had operative management. The surgical procedures performed included simple hysterectomy in twelve patients and radical hysterectomy with node dissection in eight patients. Five (5) of the 8 patients that had radical hysterectomy with node dissection and 42 patients with advanced cervical cancer were referred for radiotherapy, and only 10 returned for adjuvant chemotherapy. Squamous cell carcinoma is the commonest histological cervical cancer in our centre constituting $91.9 \%$ of all cervical cancers and $52.4 \%$ of all gynaecological malignancies. Thirty five (35) cases of ovarian cancer were seen during the period constituting $27.8 \%$ of all gynaecological malignancies with a median age of 45 years. Epithelial ovarian cancer is the commonest histological ovarian cancer and constituted $65.7 \%$ of all ovarian cancers. Majority of epithelial ovarian cancer presented in stage 3 while most of non-epithelial ovarian cancer presented in stage 1 . The uterus was the primary site of involvement in $15.9 \%$ of cases with a median age of $50.7 \pm 6.3$ years and a median age of 50 years. Five out of the twenty cases of uterine cancer, constituting $25 \%$ of uterine cancer were choriocarcinoma. Seven cases of vulva cancers were seen during the period constituting $5.6 \%$ of gynaecological malignancies and with a mean age of $67.8 \pm 2.7$ years and median age 68 years. The two cases of vagina cancer were 
seen in two 68 years old grand multiparous women. All cases of vulva and vagina cancers were squamous cell carcinoma.

The percentage incidence of gynaecological malignancies reported in our centre according to site and age group affected is presented and compared with the international data in Table 4. Compared with international data, women $<20$ years in our study did not present with gynaecological cancers (Table 4(a)). However, there was an increase in incidence of gynaecological cancers as the age increases. In Figure 1, the organ of involvement of various gynaecological malignancies was compared with data obtained from other international centers. The line graph showed that the incidence of gynaecological cancers fell in different countries from cervical to vaginal cancers. However, the incidence was lower in our center when compared with other international centers.

\section{Discussion}

Gynaecological cancers formed $13.4 \%$ of gynecological admissions in this study. This is similar when compared with $11.5 \%$ reported by Yakasai et al. in Aminu Kano University but higher than 5.4\% reported in Jos and lower when compared with $17.4 \%$ reported by Ozumba et al. in Enugu [4] [21] [23]. The profile of gynecological malignancies is different in various geographical areas [21]. Cervical cancer is one of the leading cancers in women worldwide, second only to breast

Table 4. (a) Age distribution, percentage of incidence, case by site: comparison with SEER [24]. (b) Age distribution, percentage of incidence, case by site: comparison with SEER [24].

(a)

\begin{tabular}{|c|c|c|c|c|c|c|c|c|c|c|c|c|}
\hline \multirow[t]{2}{*}{ Site } & \multicolumn{3}{|c|}{$<20$} & \multicolumn{3}{|c|}{$20-34$} & \multicolumn{3}{|c|}{$35-44$} & \multicolumn{3}{|c|}{$45-54$} \\
\hline & AEFUTHA & SEER & P Value & AEFUTHA & SEER & P Value & AEFUTHA & SEER & P Value & AEFUTHA & SEER & P Value \\
\hline Cervix & 0 & 0.2 & 1 (NS) & 12.2 & 14.5 & $<0.0001$ (sig) & 19.5 & 26.1 & 0.7885 (NS) & 14.6 & 23.7 & 0.634 (NS) \\
\hline Ovary & 0 & 1.3 & 1 (NS) & 8.3 & 3.5 & O.6742 (NS) & 12.5 & 7.4 & $<0.2478$ (NS) & $25 . \mathrm{O}$ & 19.2 & 0.2177 (NS) \\
\hline Uterus & 0 & 0.1 & 1 (NS) & 7.7 & 2.1 & 0.054 (NS) & 7.7 & 5.58 & $<0.68$ (NS) & 38.5 & 17.25 & 0.0665 (NS) \\
\hline Vulva & 0 & 1.2 & 1 (NS) & 0 & 0.9 & 1 (NS) & 0 & 5.8 & 1 (NS) & 0 & 14.0 & 1 (NS) \\
\hline Vagina & 0 & 0.2 & $1(\mathrm{NS})$ & 0 & 2.2 & $1(\mathrm{NS})$ & 0 & 8.0 & 1 (NS) & 0 & 16.2 & 1 (NS) \\
\hline
\end{tabular}

(b)

\begin{tabular}{ccccccccccc}
\hline Site & \multicolumn{3}{c}{$55-64$} & \multicolumn{3}{c}{$65-74$} & \multicolumn{3}{c}{$75-84$} \\
\hline & AEFUTHA & SEER & P Value & AEFUTHA & SEER & P Value & AEFUTHA & SEER & P Value \\
\hline Cervix & 31.7 & 16.3 & $<0.0001(\mathrm{sig})$ & 19.5 & 10.4 & $<0.001(\mathrm{sig})$ & 1.6 & 6.5 & $0.176(\mathrm{NS})$ \\
Ovary & 41.6 & 22.9 & $<0.0001(\mathrm{sig})$ & 12.5 & 19.5 & $0.5020(\mathrm{NS})$ & 0 & 18.4 & 1 (NS) \\
Uterus & 23.07 & 25.7 & $0.3198(\mathrm{NS})$ & 23.07 & 20.6 & $0.575(\mathrm{NS})$ & 0 & 17.3 & $0.109(\mathrm{NS})$ \\
Vulva & 0 & 21.7 & $0.208(\mathrm{NS})$ & 85.7 & 21.6 & $0.0002(\mathrm{sig})$ & 14.3 & 22.1 & 1 (NS) \\
Vaginal & 50.0 & 17.8 & $0.117(\mathrm{NS})$ & 50 & 17.4 & $0.11(\mathrm{NS})$ & 0 & 24.0 & 1 (NS) \\
\hline
\end{tabular}

NS = not significant, Sig = significant, SEER = surveillance, epidemiology and end result. AEFUTHA = Alex Ekwueme Federal University Teaching Hospital, Abakaliki. NB: values of AEFUTHA and SEER are expressed in percentage. 


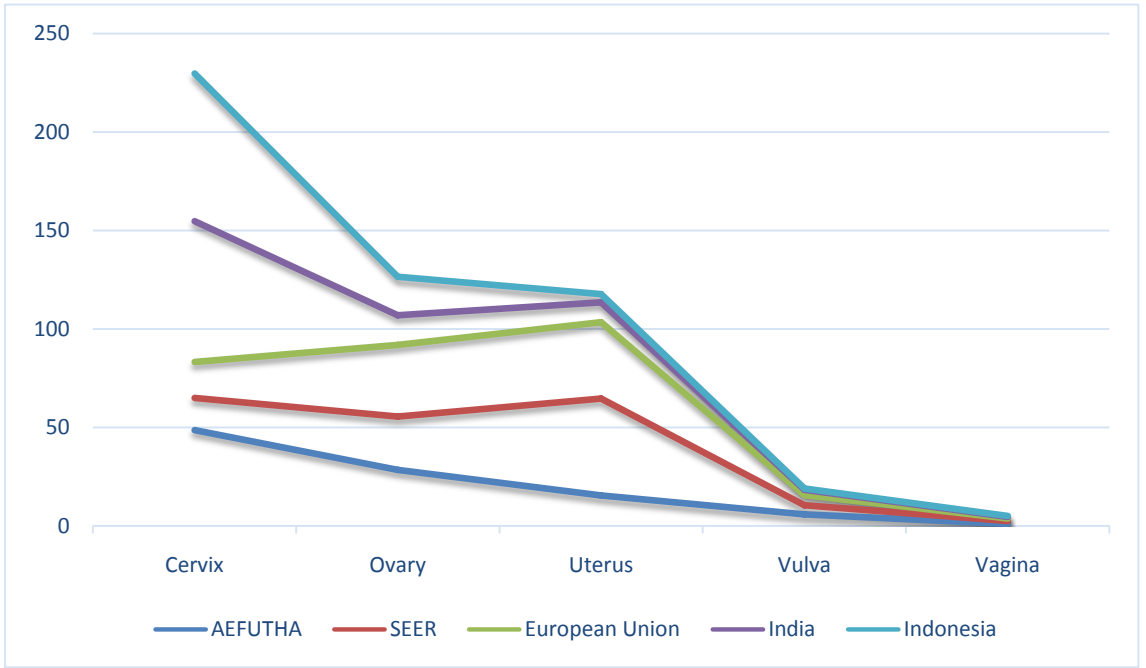

Figure 1. Comparative evaluation of site of involvement at various National Centers. AEFUTHA = Alex Ekwueme Federal University Teaching Hospital Abakaliki. SEER = Survellance Epidemiology and End Result.

cancer; $80 \%$ of new cases occur in developing countries [24]. Cervical cancer being the commonest gynaecological cancer in this study is consistent with what was reported in Northern and Eastern parts of Nigeria, India and south Asia [3] [4] [21] [23]. The proportion of cervical cancer (49.2\%), in this study, is however, low when compared with $74.9 \%$ reported in Jos and $78 \%$ reported by Ugwu et al. in Enugu but slightly higher than 48.6\% reported in Kano [3] [4] [7] [21]. Improved efforts at cervical screening and early detection of cervical dysplasia in the last 10 - 15 years in centres in developed countries have led to this reduction in the proportion of gynaecological malignancies from cervical cancer.

The position of ovarian cancer as the second commonest gynaecological cancer in this study is similar to what has been reported from Agarwal et al. in India [2], Sanni et al. in Jos [4] and in Kano by Yakasai et al. [21], but with a higher proportion of $27.8 \%$ compared to the other series [26]. The higher proportion of ovarian cancer in our study compared to other study is probably due to awareness of various gynaecological conditions created by Ministry of Health of Ebonyi state through various radio and television broadcasts, which makes women with such condition present at our centre. Besides, other peripheral hospitals have also improved in their referral systems, whereby cancers are now being referred to our centre for specialist care. However, in a study by Jamal et al. in Pakistan, ovarian tumors were the most frequent, comprising $42.4 \%$ of all gynaecological malignancies and cervical cancer, which is the most frequent in most studies in developing countries, was the second most frequent gynaecological malignancy in Pakistan [27].

Uterine malignancies are the third commonest gynaecological malignancy in this study and this was in keeping with what was recorded in other parts of the country and developing countries in India and South Asia [4] [21]. However, Surveillance, Epidemiology and End Result (SEER) in United State and studies 
done in European Union reported significantly higher proportion [24] [25]. Vulva and vagina cancers had lowest incidence in this study and this is the same across international centres. The peak age of incidence of cervical cancer was seen among 55 - 64 years age groups and this is similar to the ealier report that cervical cancer has bimodal age distribution i.e. commoner among 35 - 39 years and 60 - 64 years age groups [24]. There is also increase in incidence of cervical cancer with increasing parity which is in keeping with earlier reports [3] [4] [21] [22] [23] [24]. The relationship between increasing parity and development of ovarian, uterine, vulva and vagina cancers in this study are not statistically significant and this is similar to the earlier reports [24]. One of the important risk factors for development of malignancies is usage of tobacco. However, none of our patient gave a history of tobacco consumption. This may be due to cultural avertion to usage of tobacco in southeastern part of the country. As a result, association between tobacco usage and development of gynaecological malignancies could not be ascertained.

The median age of occurrence of cervical, vulva and vagina cancers in this study were almost the same as was observed in SEER study. However, the median age of occurrence of ovarian and uterine cancers was lower compared to SEER study [24]. A comparative analysis of proportionate site of involvement and median age was also reported. The percentage incidence of gynaecological malignancies reported in our centre according to site and age group affected is presented and compared with the international data. A comparative evaluation of data with the SEER programme revealed significantly lesser percentage of cervical malignancies among our patients in 20 - 34 years age group, whereas a higher percentage was noted in $45-64$ years age group. This may be a manifestation of better screening programme in the United State where cervical malignancies are detected in younger age group at early stage when compared with developing countries.

Among patients with uterine malignancies in this study, significant higher incidence was noted in 45 to 64 years age group. A similar trend was noticed in a report from Asian registry where uterine cancers occur a decade earlier than in the West [25]. On the contrary, relatively higher percentage of ovarian malignancies in children and young adult (from birth to 44 year) was noticed and such was similar to the findings in India [2]. The frequency of gynaecological malignancies among patients older than 75 years was low as only one case, each of cervical cancer and vulva cancer was seen in this age group and this is similar to what was reported by Agarwal et al. in India [2]. This is probably due to lower life expectancy in developing countries like Nigeria. There was no significant difference in the distribution of vaginal and vulva cancer in this study when compared with the international data [2] [24].

The extent of spread of primary malignancies in our centre was compared with data available from SEER registry [24]. Significantly higher percentage of cervical cancer presented with regional spread at our centre $(67.7 \%)$, as compared with localized disease in SEER registry cases (35.2\%). This suggested dis- 
parity in the effectiveness of screening programs. The distributions of uterine malignancy in both cohorts of patients are similar [24]. There is paucity of data on the stage of presentation and histological subtype of ovarian malignancies in African region. However, majority of ovarian cancer (68.6\%) in this study presented at advanced stage and this was similar to what was reported by SEER in which $60 \%$ of cases presented with advanced diseases, 19\% regional and 15\% with localized diseases [24], but contrary to the report of Agarwal et al. in India in which $57.14 \%$ of patients presented with localized diseases, $30.1 \%$ with regional spread and $6.63 \%$ with advanced diseases [27]. Whether this implies environmental or genetic differences in tumour profile remains to be ascertained.

This study has some limitations. Firstly, the is a hospital-based study which included only women that reach hospital but many women die of gynaecological cancers in rural areas without reaching health facility due to poverty. Community-based studies are a better tool for exploring cancer rates in developing countries. Secondly, a considerable number of women with gynaecological cancers in our centre had no histological diagnosis and were not included in this study. This probably accounted for a lower incidence of cancer types when compared to international centres.

\section{Conclusion}

This study represents a recent update on profile of gynaecological malignancies in our hospital. However due to lack of widespread population-based data, hospital-based registries form the main source of data in this country. Cervical carcinoma was the commonest gynaecological cancer in Abakaliki and majority of cases of gynaecological malignancies presented at advanced stage with its accompany treatment dilemma. There is need for a comprehensive oncology unit equipped with standardized facilities and personnel as advanced cases in this study had to be referred due to non-availability of radiotherapy equipment in our hospital. Also, there is a need for appropriate cancer registry which will enhance our policy formation and health planning.

\section{Conflicts of Interest}

The authors declare no conflict of interest.

\section{References}

[1] Mishra, K. (2011) Gynaecological Malignancies from Palliative Care Perspective. Indian Journal of Palliative Care, 17, 45-51. https://doi.org/10.4103/0973-1075.76243

[2] Agarwal, S., Malhotra, K.P., Sinha, S. and Rajaram, S. (2012) Profile of Gynaecological Malignancies Reported at a Tertiary Care Centre in India over the Past Decade: Comparative Evaluation with International Data. Indian Journal of Cancer, 48, 298-302. https://doi.org/10.4103/0019-509X.104494

[3] Moore, M.A., Ariyarante, Y., Badar, F., Bhurgri, Y., Datta, K., Matthew, A., et al. (2010) Cancer Epidermiology in South Asia-Past, Present and Future. Asian Pacific 
Journal of Cancer Prevention, 11, 49-66.

[4] Sanni, W.O., Ocheke, A.N., Oyebode, N., Jonah, M., Nyango, D.D., Silas, O.A., et al. (2013) Pattern of Gynaecological Malignancies in Jos. Tropical Journal of Obstetrics and Gynaecology, 30, 97-100.

[5] Igwegbe, A.O. and Ugboaja, J.O. (2010) Clinicopathological Analysis of Primary Ovarian Cancers in a Tertiary Health Facility in Nnewi, South Eastern Nigeria. Nigerian Medical Practitioner, 57, 27-30. https://doi.org/10.4314/nmp.v57i3.55749

[6] Fleming, N.D. and Dorigo, O.D. (2013) Premalignant and Malignant Disorders of the Uterine Corpus. In: Current Diagnosis and Treatment: Obstetrics and Gynaecology, 11th Edition, The McGraw-Hill Companies, New York, 832-847.

[7] Ugwu, E.O., Iferikigwe, E.S., Okeke, T.C., Ugwu, A.O., Okezie, O.A. and Agu, P.U. (2011) Pattern of Gynaecological Cancers in University of Nigeria Teaching Hospital, Enugu, South Eastern Nigeria. Nigerian Journal Medicine, 20, 266-269.

[8] The Economist Intelligence Unit (2009) Breakaway: The Global Burden of Cancer-Challenges and Opportunities. The Economist.

https://www.economist.com/subciption

[9] Kaiser Family Foundation Fast Facts. https://www.globalhealthfacts.org

[10] Cancer Research United Kingdom 2013. https://www.cancerresearchuk.org $>$ cs

[11] Iyoke, C.A. and Ugwu, G.O. (2013) Burden of Gynaecological Cancer in Developing Countries. World Journal of Obstetrics and Gynecology, 2, 1-7. https://doi.org/10.5317/wjog.v2.i1.1

[12] Price, A.J., Ndom, P., Atenguena, E., Nouemssi, J.P.M. and Ryder, R.W. (2011) Cancer Care Challenges in Developing Countries. Cancer, 118, 3627-3635. https://doi.org/10.1002/cncr.26681

[13] Sarkar, M., Konar, H. and Raut, D. (2013) Clinico-Pathological Features of Gynaecological Malignancies in a Tertiary Care Hospital in Eastern India: Importance of Strengthening Primary Health Care in Prevention and Early Detection. Asian Pacific Journal of Cancer Prevention, 14, 3541-3547. https://doi.org/10.7314/APJCP.2013.14.6.3541

[14] Pathy, S., Raheja, S.J. and Rakh, S. (2008) Pain in Advanced Gynaecological Malignancies: Institute of Palliative Care Experience. Indian Journal of Palliative Care, 14, 86-89. https://doi.org/10.4103/0973-1075.45451

[15] Sarkar, M., Konar, H. and Raut, D.K. (2012) Gynaecological Malignancies: Epidemiological Characteristics of the Patients in a Tertiary Care Hospital in India. Asian Pacific Journal of Cancer Prevention, 13, 2997-3004. https://doi.org/10.7314/APJCP.2012.13.6.2997

[16] Sarkar, M., Konar, H. and Raut, D.K. (2011) Knowledge and Health Care-Seeking Behavior in Relation to Gynaecological Malignancies in India: A Study of the Patients with Gynaecological Malignancies in a Tertiary Care Hospital of Kolkata. Journal of Cancer Education, 26, 348-354. https://doi.org/10.1007/s13187-010-0168-9

[17] Hemalatha, A.L., Gayathri, M.N., Deepthi, B.R., Neelima, P.C., Giripunja, M. and Nayana, N.S. (2013) Evaluation of Trends in the Profile of Gynaecological Malignancies at a Tertiary Care Hospital in Karnataka, South India. International Journal of Medical Research and Health Sciences, 2, 870-873.

https://doi.org/10.5958/j.2319-5886.2.4.139 
[18] Momtahen, S., Kadivar, M., Kazzazi, A.S. and Gholipour, F. (2009) Assessment of Gynaecologic Malignancies: A Multi-Center Study in Tehran (1995-2005). Indian Journal of Cancer, 46, 226-230. https://doi.org/10.4103/0019-509X.52957

[19] Laryea, D.O., Awuah, B., Amoako, Y.A., Osei-Bonsu, E., Dogbe, J., Larsen-Reindorf, R., et al. (2014) Cancer Incidence in Ghana, 2012: Evidence from a Population-Based Cancer Registry. BMC Cancer, 14, 362. https://doi.org/10.1186/1471-2407-14-362

[20] Luesley, D.M. (2012) Malignant Diseases of the Vulva and the Vagina. In: Dewhurst's Textbook of Obstetrics and Gynaecology, 8th Ed, John Wiley and Son, Ltd., New York, 729-745. https://doi.org/10.1002/9781119979449.ch55

[21] Yakasai, I.A., Ugwa, E.A. and Otubu, J. (2013) Gynaecological Malignancies in Aminu Kano Teaching Hospital Kano: A 3 Year Review. Nigerian Journal of Clinical Practice, 16, 63-66. https://doi.org/10.4103/1119-3077.106768

[22] Iyoke, C.A., Ugwu, G.O., Ezugwu, E.C., Ezugwu, F.O., Onyebuchi, A.K. and Lawani, O.L. (2014) Challenges Associated with the Management of Gynaecological Cancers in a Tertiary Hospital in South East Nigeria. International Journal of Women's Health, 6, 123-130. https://doi.org/10.2147/IJWH.S55797

[23] Ozumba, B.C., Nzegwu, A.M. and Anyikam, A. (2011) Histological Patterns of Gynaecological Lesions in Enugu, Nigeria. A Five-Year Review from January 1, 2000 to December 31st 2004. Advances in Bioresearch, 2, 132-136.

[24] Altekruse, S.F., Kosary, C.L., Krapcho, M., Neyman, N., Aminou, R., Waldron, W., Ruhl, J., Howlader, N., Tatalovich, Z., Cho, H., Mariotto, A., Eisner, M.P., Lewis, D.R., Cronin, K., Chen, H.S., Feuer, E.J., Stinchcomb, D.G. and Edwards, B.K. (2007) SEER Cancer Statistics Review, 1975-2007. North American Association of Cancer Registries, Bethesda, MD, c2007. https://www.seer.cancer.gov

[25] Boyle, P. and Ferlay, J. (2005) Cancer Incidence and Mortality in Europe, 2004. Annals of Oncology, 16, 481-88. https://doi.org/10.1093/annonc/mdi098

[26] Aziz, M.F. (2009) Gynaecological Cancer in Indonesia. Journal of Gynaecological Oncology, 20, 8-10. https://doi.org/10.3802/jgo.2009.20.1.8

[27] Jamal, S., Mamoon, N., Mushtaq, S., Luqman, M. and Moghal, S. (2006) The Pattern of Gynaecological Malignancies in 968 Cases from Pakistan. Annals of Saudi Medicine, 26, 382-384. https://doi.org/10.5144/0256-4947.2006.382 